\title{
A Sub-Supersolution Approach for Robin Boundary Value Problems with Full Gradient Dependence
}

\author{
Dumitru Motreanu ${ }^{1, *}$, Angela Sciammetta ${ }^{2}(\mathbb{D}$ and Elisabetta Tornatore $2(\mathbb{D}$ \\ 1 Department of Mathematics, University of Perpignan, 66860 Perpignan, France \\ 2 Department of Mathematics and Computer Science, University of Palermo, 90123 Palermo, Italy; \\ angela.sciammetta@unipa.it (A.S.); elisa.tornatore@unipa.it (E.T.) \\ * Correspondence: motreanu@univ-perp.fr
}

Received: 19 March 2020; Accepted: 19 April 2020; Published: 27 April 2020

\begin{abstract}
The paper investigates a nonlinear elliptic problem with a Robin boundary condition, which exhibits a convection term with full dependence on the solution and its gradient. A subsupersolution approach is developed for this type of problems. The main result establishes the existence of a solution enclosed in the ordered interval formed by a sub-supersolution. The result is applied to find positive solutions.
\end{abstract}

Keywords: nonlinear elliptic problem; Robin boundary condition; gradient dependence; sub-supersolution; positive solution

\section{Introduction}

In this paper we study the following nonlinear elliptic boundary value problem

$$
\begin{cases}-\operatorname{div}(A(x, \nabla u))+\alpha(x)|u|^{p-2} u=f(x, u, \nabla u) & \text { in } \Omega \\ A(x, \nabla u) \cdot v(x)+\beta(x)|u|^{p-2} u=0 & \text { on } \partial \Omega\end{cases}
$$

on a bounded domain $\Omega \subset \mathbb{R}^{N}$ with $N \geq 3$ and with a boundary $\partial \Omega$ of class $C^{1}$. The notation $v(x)$ stands for the unit exterior normal at any $x \in \partial \Omega$ and $p$ is a real number with $1<p<+\infty$. We note that, in the stated problem, the boundary condition is of Robin type.

We describe the data entering our problem. The leading differential part of the equation in (1) is the term $\operatorname{div}(A(x, \nabla u))$ in divergence form driven by the map $A: \bar{\Omega} \times \mathbb{R}^{N} \rightarrow \mathbb{R}^{N}$ which is composed with the (weak) gradient $\nabla u$ of the solution $u: \Omega \rightarrow \mathbb{R}$. No homogeneity condition is required for the map $A$. Precisely, we assume that $A: \bar{\Omega} \times \mathbb{R}^{N} \rightarrow \mathbb{R}^{N}$ is continuous and fulfills the conditions:

(A1) There exist constants $c_{1}$ and $c_{2}$ with $0<c_{1} \leq c_{2}$ such that

$$
A(x, \xi) \cdot \xi \geq c_{1}|\xi|^{p} \text { and }|A(x, \xi)| \leq c_{2}\left(|\xi|^{p-1}+1\right) \text { for all }(x, \xi) \in \bar{\Omega} \times \mathbb{R}^{N} .
$$

(A2) For all $x \in \Omega, A(x, \xi)$ is strictly monotone in $\xi$.

Here and subsequently we denote by $|\cdot|$ and $\cdot$ the standard Euclidean norm and scalar product on $\mathbb{R}^{N}$, respectively.

As important examples of operators $\operatorname{div}(A(x, \nabla u))$ complying with the preceding hypotheses we mention: the $p$-Laplacian $\Delta_{p} u:=\operatorname{div}\left(|\nabla u|^{p-2} \nabla u\right)$ where $A(x, \xi)=|\xi|^{p-2} \xi$, the $(p, q)$-Laplacian $\Delta_{p} u+\Delta_{q} u:=\operatorname{div}\left(\left(|\nabla u|^{p-2}+|\nabla u|^{q-2}\right) \nabla u\right)$ where $1<q<p<+\infty$ and $A(x, \xi)=|\xi|^{p-2} \xi+|\xi|^{q-2} \xi$, the generalized $p$-mean curvature operator $\operatorname{div}\left(\left(1+|\nabla u|^{2}\right)^{\frac{p-2}{2}} \nabla u\right)$ where $A(x, \xi)=\left(1+|\xi|^{2}\right)^{\frac{p-2}{2}}$ as well as numerous weighted versions. 
The values of $u$ on $\partial \Omega$ in the boundary condition of (1) are in the trace sense, whereas $A(x, \nabla u)$. $v(x)$ represents the co-normal derivative of $u$ associated with $A$. For more details we refer to ([1], pages 7-9) and ([2], Section 2). In the statement of problem (1) we fix the functions $\alpha \in L^{\infty}(\Omega)$ and $\beta \in L^{\infty}(\partial \Omega)$ satisfying $\alpha(x) \geq 0$ for almost everywhere (in short a.e.) $x \in \Omega$ and $\beta(x) \geq 0$ for a.e. $x \in \partial \Omega, \beta \neq \equiv$, where $\partial \Omega$ is endowed with the $(N-1)$-dimensional Hausdorff measure. Contrary to the Neumann problem, here it is allowed to have $\alpha=0$. Recall that if $\alpha \in L^{\infty}(\Omega)$ with $\alpha \geq 0, \alpha \not \equiv 0$, the term $\alpha(x)|u|^{p-2} u$ was essential to develop the method of sub-supersolution under Neumann boundary condition (see [3]). Actually, in the Robin problem, the hypothesis $\beta(x) \geq 0$ for a.e. $x \in \partial \Omega, \beta \not \equiv 0$, is a substitute for the condition $\alpha(x) \geq 0$ for a.e. $x \in \Omega, \alpha \neq \equiv$, assumed for the Neumann problem.

The reaction term $f(x, u, \nabla u)$ in the equation (1) is determined by a Carathéodory function $f: \Omega \times \mathbb{R} \times \mathbb{R}^{N} \rightarrow \mathbb{R}$, i.e., $f(\cdot, s, \xi)$ is measurable for all $(s, \xi) \in \mathbb{R} \times \mathbb{R}^{N}$ and $f(x, \cdot, \cdot)$ is continuous for a.e. $x \in \Omega$. This term, depending not only on the solution $u$ but also on its gradient $\nabla u$, is called convection. It prevents to have a variational structure for problem (1) and thus the variational methods are not applicable, which creates a serious difficulty for handling (1).

The Robin problems exhibiting convection term as is the case in (1) have only recently been studied. We refer to [4-8] for results on the existence of solutions to such problems, where the approach is based on fixed point theorems or on surjectivity criteria for monotone-type operators. We also mention that a singular Robin problem involving convection has recently been treated in [9]. There are many results for Robin problems with variational structure, thus without a convection term. In this direction, we cite, e.g., [10-15]. The aim of the present work is to study the Robin problem (1) with general gradient dependence through the method of sub-supersolution. Due to the lack of variational structure, one cannot handle such a problem by variational methods. We recall that in the study of non-variational elliptic problems one develops arguments as, for instance, the lower and upper solution method with monotone iterations, approximation approach of Galerkin-type, surjectivity theorems for monotone-type operators, fixed point theorems, topological degree theory, bifurcation theory examining phenomena as branches of solutions and blow-up. It is beyond the scope of our paper to review this huge amount of work. We only illustrate certain of these topics with a few recent references: a comparison principle and approximation process relying on a Schauder basis in [16], a fixed point approach using minimal solutions in [17], estimates based on Trudinger-Moser inequality for problems with exponential nonlinearities in [18]. We also mention the classical monographs [19,20], which are fundamental references for general elliptic equations.

According to our knowledge, this is the first time when the method of sub-supersolution is systematically implemented for nonlinear Robin problems with convection. We prove a general existence and location result for a solution to be enclosed in the ordered interval determined by a sub-supersolution. Specifically, given a subsolution $\underline{u}$ and a supersolution $\bar{u}$ for problem (1) with $\underline{u} \leq \bar{u}$ a.e. in $\Omega$ (see Section 2 for the relevant definitions), our main abstract result provides the existence of a solution $u$ to problem (1) satisfying $\underline{u} \leq u \leq \bar{u}$ a.e. in $\Omega$. This is an important qualitative property of the solution $u$ offering a priori estimates. The growth condition that we suppose in the variable $s$ for the nonlinearity $f(x, s, \xi)$ concerns only the real interval $[\underline{u}(x), \bar{u}(x)]$. We emphasize that our abstract result can be applied provided we know sub-supersolutions, i.e., ordered pairs of a subsolution $\underline{u}$ and a supersolution $\bar{u}$ for problem (1) with $\underline{u} \leq \bar{u}$ i.e., in $\Omega$, so the task to find such ordered pairs becomes the primary task in applying the method. In this sense, we provide an application of our main result to get positive solutions for a class of nonlinear Robin problem with convection term by showing explicitly how one can effectively determine sub-supersolutions. Results, as are given here, have recently been established in [21] for nonlinear Dirichlet problems with convection and in [3] for nonlinear Neumann problems with convection. General ideas regarding the method of sub-supersolution can be found in $[1,22]$.

The rest of the paper is organized as follows. Section 2 discusses the background needed in the sequel. Section 3 focuses on a related operator equation, which is of independent interest. Section 4 sets forth our main result. Section 5 contains our application to produce positive solutions. 


\section{Prerequisites of Sub-Supersolution Method}

This section contains preliminaries that will be used in the sequel. First, we fix some notation. For any $r \in \mathbb{R}$, we set $r^{+}=\max \{r, 0\}$ (the positive part of $r$ ). If $r>1$, we also set $r^{\prime}=\frac{r}{r-1}$ (the Hölder conjugate of $r$ ). In particular, for $p \in(1,+\infty)$ we have $p^{\prime}=\frac{p}{p-1}$.

As indicated in Section $1, \Omega$ is a bounded domain in $\mathbb{R}^{N}$ with $N \geq 3$ whose boundary $\partial \Omega$ is of class $C^{1}$. In order to avoid repetitive arguments, we suppose that $N>p$. The complementary case $N \leq p$ can be treated along the same lines and actually is easier. By $\|\cdot\|_{L^{r}(\Omega)}$ we denote the usual norm on the Banach space $L^{r}(\Omega)$.

We seek the solutions to problem (1) in the Sobolebv space $W^{1, p}(\Omega)$, which is a Banach space equipped with the norm

$$
\|u\|_{1, p}:=\left(\|u\|_{L^{p}(\Omega)}^{p}+\|\nabla u\|_{L^{p}(\Omega)}^{p}\right)^{\frac{1}{p}} .
$$

For our study of problem (1) it is convenient to use the following equivalent norm on $W^{1, p}(\Omega)$ (see, e.g., ([23], Lemma 2.7) or ([15], Proposition 2.8))

$$
\|u\|_{\beta, 1, p}:=\left(\int_{\partial \Omega} \beta(\sigma)|u(\sigma)|^{p} d \sigma+\|\nabla u\|_{L^{p}(\Omega)}^{p}\right)^{\frac{1}{p}} .
$$

The dual space of $W^{1, p}(\Omega)$ is denoted $\left(W^{1, p}(\Omega)\right)^{*}$, while the notation $\langle\cdot, \cdot\rangle$ designates the duality pairing between $W^{1, p}(\Omega)$ and $\left(W^{1, p}(\Omega)\right)^{*}$, we denote by $\rightarrow$ the strong convergence and by $\rightarrow$ the weak convergence. The Sobolev embedding theorem ensures that the space $W^{1, p}(\Omega)$ is continuously embedded in $L^{p^{*}}(\Omega)$, where $p^{*}$ is the Sobolev critical exponent $p^{*}=\frac{N p}{N-p}$ (we have supposed $N>p$ ). Moreover, by the Rellich-Kondrachov theorem, $W^{1, p}(\Omega)$ is compactly embedded in $L^{r}(\Omega)$ for every $r \in\left[1, p^{*}\right)$.

Corresponding to the map $A: \bar{\Omega} \times \mathbb{R}^{N} \rightarrow \mathbb{R}^{N}$ describing the principal part of the equation in problem (1), we introduce the operator $\tilde{A}: W^{1, p}(\Omega) \rightarrow\left(W^{1, p}(\Omega)\right)^{*}$ defined by

$$
\langle\tilde{A}(u), v\rangle=\int_{\Omega} A(x, \nabla u) \cdot \nabla v d x \text { for all } u,
$$

which is well defined thanks to assumption $(A 1)$. It turns out from assumption $(A 2)$ and the continuity of $A$ that $A(x, \xi)$ is maximal monotone in the variable $\xi$ for all $x \in \Omega$. This allows us to invoke ([2], Proposition 10), which yields:

Proposition 1. Assume that the continuous map $A: \bar{\Omega} \times \mathbb{R}^{N} \rightarrow \mathbb{R}^{N}$ satisfies the conditions (A1) and $(A 2)$. Then the map $\tilde{A}: W^{1, p}(\Omega) \rightarrow\left(W^{1, p}(\Omega)\right)^{*}$ in (3) has the $\left(S_{+}\right)$-property, that is, any sequence $\left\{u_{n}\right\} \subset W^{1, p}(\Omega)$ with $u_{n} \rightarrow u$ in $W^{1, p}(\Omega)$ and $\limsup _{n \rightarrow+\infty}\left\langle\tilde{A}\left(u_{n}\right), u_{n}-u\right\rangle \leq 0$ fulfills $u_{n} \rightarrow u$ in $W^{1, p}(\Omega)$.

There exists a unique continuous linear map $\gamma: W^{1, p}(\Omega) \rightarrow L^{p}(\partial \Omega)$ called the trace map such that

$$
\gamma(u)=u_{\mid \partial \Omega} \text { for all } u \in W^{1, p}(\Omega) \cap C(\bar{\Omega}) .
$$

The kernel of $\gamma: W^{1, p}(\Omega) \rightarrow L^{p}(\partial \Omega)$ is $W_{0}^{1, p}(\Omega)$. Recalling that $N>p$, the trace map $\gamma$ is compact from $W^{1, p}(\Omega)$ into $L^{\eta}(\partial \Omega)$ for all $\eta \in\left[1, \frac{(N-1) p}{N-p}\right)$ (see, e.g., ([22], Theorem 2.79)). As usual, we drop the notation of the trace map $\gamma$ writing simply $u$ in place of $\gamma(u)$. The co-normal derivative $A(x, \nabla u) \cdot v(x)$, appearing in the boundary condition in problem (1), is obtained by extending the map $u(\cdot) \mapsto A(\cdot, \nabla u(\cdot)) \cdot v(\cdot)$, from $C^{1}(\bar{\Omega})$ to $W^{1, p}(\Omega)$. 
By a (weak) solution to problem (1) we mean a function $u \in W^{1, p}(\Omega)$ such that $f(x, u, \nabla u) \in$ $L^{\left(p^{*}\right)^{\prime}}(\Omega)$ and

$$
\int_{\Omega} A(x, \nabla u) \cdot \nabla v d x+\int_{\Omega} \alpha(x)|u|^{p-2} u v d x+\int_{\partial \Omega} \beta(x)|u|^{p-2} u v d \sigma=\int_{\Omega} f(x, u, \nabla u) v d x
$$

for all $v \in W^{1, p}(\Omega)$.

A function $\underline{u} \in W^{1, p}(\Omega)$ is called a subsolution for problem $(1)$ if $f(\cdot, \bar{u}(\cdot), \nabla \bar{u}(\cdot)) \in L^{\left(p^{*}\right)^{\prime}}(\Omega)$ and

$$
\int_{\Omega}\left(A(x, \nabla \underline{u}) \cdot \nabla v+\alpha(x)|\underline{u}|^{p-2} \underline{u} v\right) d x+\int_{\partial \Omega} \beta(x)|\underline{u}|^{p-2} \underline{u} v d \sigma \leq \int_{\Omega} f(x, \underline{u}, \nabla \underline{u}) v d x,
$$

for all $v \in W^{1, p}(\Omega), v \geq 0$ a.e. in $\Omega$.

Symmetrically, a function $\bar{u} \in W^{1, p}(\Omega)$ is called a supersolution for problem (1) if $f(\cdot, \bar{u}(\cdot), \nabla \bar{u}(\cdot)) \in L^{\left(p^{*}\right)^{\prime}}(\Omega)$ and

$$
\int_{\Omega}\left(A(x, \nabla \bar{u}) \cdot \nabla v+\alpha(x)|\bar{u}|^{p-2} \bar{u} v\right) d x+\int_{\partial \Omega} \beta(x)|\bar{u}|^{p-2} \bar{u} v d \sigma \geq \int_{\Omega} f(x, \bar{u}, \nabla \bar{u}) v d x,
$$

for all $v \in W^{1, p}(\Omega), v \geq 0$ a.e. in $\Omega$.

Due to assumption $(A 1)$, the integrals in the above definitions exist. We notice that $u \in W^{1, p}(\Omega)$ is a solution of (1) if and only if $u$ is simultaneously a subsolution and a supersolution.

We are going to argue with a sub-supersolution for problem (1), that is, an ordered pair of a subsolution $\underline{u}$ and a supersolution $\bar{u}$ such that $\underline{u} \leq \bar{u}$, which means the pointwise inequality $\underline{u}(x) \leq \bar{u}(x)$ for a.e. $x \in \Omega$. Then we can associate the ordered interval

$$
[\underline{u}, \bar{u}]=\left\{w \in W^{1, p}(\Omega): \underline{u} \leq w \leq \bar{u}\right\}
$$

Our goal is to obtain a solution $u \in W^{1, p}(\Omega)$ of problem (1) with the location property $u \in[\underline{u}, \bar{u}]$, which will be achieved through comparison by means of a truncation operator that we now describe. Corresponding to a subsolution $\underline{u}$ and a supersolution $\bar{u}$ satisfying $\underline{u} \leq \bar{u}$ a.e. in $\Omega$, we define the truncation operator $T=T(\underline{u}, \bar{u}): W^{1, p}(\Omega) \rightarrow W^{1, p}(\Omega)$ by

$$
T(u)(x)=\left\{\begin{array}{lll}
\underline{u}(x) & \text { if } & u(x)<\underline{u}(x) \\
u(x) & \text { if } & \underline{u}(x) \leq \bar{u}(x) \leq \bar{u}(x) \\
\bar{u}(x) & \text { if } & u(x)>\bar{u}(x)
\end{array}\right.
$$

for all $u \in W^{1, p}(\Omega)$ and a.e. $x \in \Omega$. It readily follows that $T: W^{1, p}(\Omega) \rightarrow W^{1, p}(\Omega)$ is continuous and bounded (in the sense that it maps bounded sets into bounded sets).

We shall also need the (negative) Dirichlet $p$-Laplacian, which is the operator $-\Delta_{p}: W_{0}^{1, p}(\Omega) \rightarrow$ $W^{-1, p^{\prime}}(\Omega)=\left(W_{0}^{1, p}(\Omega)\right)^{*}$ given by

$$
\left\langle-\Delta_{p} u, v\right\rangle=\int_{\Omega}|\nabla u|^{p-2} \nabla u \cdot \nabla v d x \text { for all } u, v \in W_{0}^{1, p}(\Omega)
$$

It is well-known (see, e.g., ([1], Proposition 9.47)) that there exists a least positive number $\lambda_{1}>0$ (called the first eigenvalue of $-\Delta_{p}$ ) for which the Dirichlet problem

$$
\begin{cases}-\Delta_{p} \varphi_{1}=\lambda_{1}\left|\varphi_{1}\right|^{p-2} \varphi_{1} & \text { in } \Omega \\ \varphi_{1}=0 & \text { on } \partial \Omega\end{cases}
$$

has a nontrivial solution $\varphi_{1} \in W_{0}^{1, p}(\Omega)$. By the regularity theory we have $\varphi_{1} \in C^{1}(\bar{\Omega})$. Moreover, we can choose $\varphi_{1}$ to satisfy $\varphi_{1}>0$ in $\Omega$. 
Finally, we mention a few things about the pseudomonotone operators. Let $X$ be a Banach space with the norm $\|\cdot\|$ and its dual $X^{*}$. We denote by $\langle\cdot, \cdot\rangle$ the duality pairing between $X$ and $X^{*}$. A map $\mathcal{A}: X \rightarrow X^{*}$ is called bounded if it maps bounded sets into bounded sets. The map $\mathcal{A}: X \rightarrow X^{*}$ is said to be coercive if

$$
\lim _{\|u\| \rightarrow+\infty} \frac{\langle\mathcal{A}(u), u\rangle}{\|u\|}=+\infty .
$$

The map $\mathcal{A}: X \rightarrow X^{*}$ is called pseudomonotone if for each sequence $\left(u_{n}\right) \subset X$ satisfying $u_{n} \rightarrow u$ in $X$ and $\lim \sup _{n \rightarrow \infty}\left\langle\mathcal{A}\left(u_{n}\right), u_{n}-u\right\rangle \leq 0$, it holds

$$
\langle\mathcal{A}(v), u-v\rangle \leq \liminf _{n \rightarrow \infty}\left\langle\mathcal{A}\left(u_{n}\right), u_{n}-v\right\rangle \text { for all } v \in X .
$$

The main theorem for pseudomonotone operators reads as follows (see, e.g., ([22], Theorem 2.99)).

Theorem 1. Let $X$ be a reflexive Banach space. If $\mathcal{A}: X \rightarrow X^{*}$ is a pseudomonotone, bounded and coercive map, then $\mathcal{A}$ is surjective.

\section{The Associated Operator Equation}

Assume that a subsolution $\underline{u}$ and a supersolution $\bar{u}$ for problem (1) with $\underline{u} \leq \bar{u}$ are given and that $f: \Omega \times \mathbb{R} \times \mathbb{R}^{N} \rightarrow \mathbb{R}$ satisfies the following growth condition adapted to the ordered interval $[\underline{u}, \bar{u}]:$

$(H)$ There exist a function $\sigma \in L^{r^{\prime}}(\Omega)$ with $r \in\left(1, p^{*}\right)$ and constants $a>0$ and $r_{1} \in\left(0, \frac{p}{\left(p^{*}\right)^{\prime}}\right)$ such that

$$
|f(x, s, \xi)| \leq \sigma(x)+a|\xi|^{r_{1}} \text { for a.e. } x \in \Omega, \text { all } s \in[\underline{u}(x), \bar{u}(x)], \xi \in \mathbb{R}^{N}
$$

We introduce the cut-off function $\pi: \Omega \times \mathbb{R} \rightarrow \mathbb{R}$ defined by

$$
\pi(x, s)= \begin{cases}-(\underline{u}(x)-s)^{\frac{r_{1}}{p-r_{1}}} & \text { if } \quad s<\underline{u}(x), \\ 0 & \text { if } \quad \underline{u}(x) \leq s \leq \bar{u}(x), \\ (s-\bar{u}(x))^{\frac{r_{1}}{p-r_{1}}} & \text { if } \quad s>\bar{u}(x),\end{cases}
$$

where $r_{1}>0$ is the constant postulated in hypothesis $(H)$. From $(9)$ and the fact that $\underline{u}, \bar{u} \in L^{p^{*}}(\Omega)$ we infer that $\pi$ verifies the growth condition

$$
|\pi(x, s)| \leq c|s|^{\frac{r_{1}}{p-r_{1}}}+\varrho(x) \text { for a.e. } x \in \Omega, \text { all } s \in \mathbb{R},
$$

with a constant $c>0$ and a function $\varrho \in L^{\frac{p^{*}\left(p-r_{1}\right)}{r_{1}}}(\Omega)$.

Now for every $\lambda>0$ we define the nonlinear operator $A_{\lambda}: W^{1, p}(\Omega) \rightarrow\left(W^{1, p}(\Omega)\right)^{*}$ by

$$
\begin{aligned}
\left\langle A_{\lambda}(u), v\right\rangle & =\int_{\Omega} A(x, \nabla u) \cdot \nabla v d x+\int_{\Omega} \alpha(x)|u|^{p-2} u v d x+\int_{\partial \Omega} \beta(x)|u|^{p-2} u v d \sigma \\
& +\lambda \int_{\Omega} \pi(x, u) v d x-\int_{\Omega} f(x, T u, \nabla T u) v d x \text { for all } u, v \in W^{1, p}(\Omega) .
\end{aligned}
$$

Hypothesis $(H)$ guarantees that the operator $A_{\lambda}$ in (11) is well defined.

Due to (10), we may consider the Nemytskij operator $\Pi: L^{p^{*}}(\Omega) \rightarrow L^{\frac{p^{*}\left(p-r_{1}\right)}{r_{1}}}(\Omega)$, associated to the function $\pi$ in (10), namely $\Pi(u)=\pi(\cdot, u(\cdot))$ for all $u \in L^{p^{*}}(\Omega)$. It is well defined, continuous and bounded. The condition in $(H)$ that $r_{1}<\frac{p}{\left(p^{*}\right)^{\prime}}$ is equivalent to $\frac{p^{*}\left(p-r_{1}\right)}{r_{1}}>\left(p^{*}\right)^{\prime}$. Hence, by the Rellich-Kondrachov compact embedding theorem, the Nemytskij operator $\Pi: W^{1, p}(\Omega) \rightarrow$ $\left(W^{1, p}(\Omega)\right)^{*}$ is completely continuous. 
Thanks to hypothesis $(H)$ we also have the Nemytskij operator $N_{f}:[\underline{u}, \bar{u}] \rightarrow\left(W^{1, p}(\Omega)\right)^{*}$ on the ordered interval $[\underline{u}, \bar{u}]$ which is associated to the function $f: \Omega \times \mathbb{R} \times \mathbb{R}^{N} \rightarrow \mathbb{R}$, that is

$$
\left\langle N_{f}(u), v\right\rangle=\int_{\Omega} f(x, u(x), \nabla u(x)) v(x) d x
$$

for all $u \in[u, \bar{u}]$ and $v \in W^{1, p}(\Omega)$. Using $(H)$ we see that $f(\cdot, u(\cdot), \nabla u(\cdot)) \in L^{\frac{p}{r_{1}}}(\Omega)$. As $v \in$ $L^{p^{*}}(\Omega)$ and $\frac{p}{r_{1}}>\left(p^{*}\right)^{\prime}$, the above integral exists. By virtue of the strict inequality $\frac{p}{r_{1}}>\left(p^{*}\right)^{\prime}$, the Rellich-Kondrachov compact embedding theorem implies that the Nemytskij operator $N_{f}:[\underline{u}, \bar{u}] \rightarrow$ $\left(W^{1, p}(\Omega)\right)^{*}$ is completely continuous.

Again through the Rellich-Kondrachov compact embedding theorem we can show that the operator $B: W^{1, p}(\Omega) \rightarrow\left(W^{1, p}(\Omega)\right)^{*}$ given by

$$
\langle B(u), v\rangle=\int_{\Omega} \alpha(x)|u(x)|^{p-2} u(x) v(x) d x
$$

for all $u, v \in W^{1, p}(\Omega)$ is completely continuous.

Consider also the operator $\Gamma: W^{1, p}(\Omega) \rightarrow\left(W^{1, p}(\Omega)\right)^{*}$ given by

$$
\langle\Gamma(u), v\rangle=\int_{\partial \Omega} \beta(\sigma)|u(\sigma)|^{p-2} u(\sigma) v(\sigma) d \sigma
$$

for all $u, v \in W^{1, p}(\Omega)$, where the integration is done with respect to the $(N-1)$-dimensional Hausdorff (surface) measure on $\partial \Omega$.

Let us check that the map $\Gamma: W^{1, p}(\Omega) \rightarrow\left(W^{1, p}(\Omega)\right)^{*}$ is completely continuous. To this end, let $u_{n} \rightarrow u$ in $W^{1, p}(\Omega)$. Then the compactness of the trace map $\gamma: W^{1, p}(\Omega) \rightarrow L^{p}(\partial \Omega)$ ensures the strong convergence $u_{n} \equiv \gamma\left(u_{n}\right) \rightarrow u \equiv \gamma(u)$ in $L^{p}(\partial \Omega)$, thus the strong convergence $\left|u_{n}\right|^{p-2} u_{n} \rightarrow|u|^{p-2} u$ in $L^{p^{\prime}}(\partial \Omega)$. Taking into account $(12)$ we deduce that $\Gamma\left(u_{n}\right) \rightarrow \Gamma(u)$ in $\left(W^{1, p}(\Omega)\right)^{*}$, so $\Gamma: W^{1, p}(\Omega) \rightarrow$ $\left(W^{1, p}(\Omega)\right)^{*}$ is completely continuous.

For every $\lambda>0$, the operator $A_{\lambda}: W^{1, p}(\Omega) \rightarrow\left(W^{1, p}(\Omega)\right)^{*}$ in (11) has the expression

$$
A_{\lambda}=\tilde{A}+B+\Gamma+\lambda \Pi-N_{f} \circ T .
$$

The composition $N_{f} \circ T$ makes sense because $T$ takes values in the ordered interval $[\underline{u}, \bar{u}]$ as seen from (7). The following theorem asserts the solvability of the equation

$$
A_{\lambda}(u)=0 .
$$

Theorem 2. Assume that the conditions (A1), $(A 2)$ and $(H)$ are satisfied. Then Equation (14) possesses at least a solution $u \in W^{1, p}(\Omega)$ provided $\lambda>0$ is sufficiently large.

Proof. In order to prove the solvability of operator Equation (14) we apply Theorem 1. We have to prove that the operator $A_{\lambda}: W^{1, p}(\Omega) \rightarrow\left(W^{1, p}(\Omega)\right)^{*}$ in (13) is bounded, pseudomonotone and coercive.

By (3) and hypothesis $(A 1)$, in conjunction with Hölder's inequality and the Sobolev embedding theorem, we find that 


$$
\begin{aligned}
\|\tilde{A}(u)\|_{\left(W^{1, p}(\Omega)\right)^{*}}^{p} & =\sup _{\|v\|_{\beta, 1, p} \leq 1}|\langle\tilde{A}(u), v\rangle| \\
& =\sup _{\|v\|_{\beta, 1, p} \leq 1}\left|\int_{\Omega} A(x, \nabla u) \cdot \nabla v d x\right| \\
& \leq c_{2} \sup _{\|v\|_{\beta, 1, p} \leq 1} \int_{\Omega}\left(|\nabla u|^{p-1}+1\right)|\nabla v| d x \\
& \leq C\left(\|u\|_{\beta, 1, p}^{p-1}+1\right)
\end{aligned}
$$

for all $u \in W^{1, p}(\Omega)$, with a constant $C>0$. This shows that the operator $\tilde{A}: W^{1, p}(\Omega) \rightarrow\left(W^{1, p}(\Omega)\right)^{*}$ is bounded.

The composed operator $N_{f} \circ T$ is bounded because $T$ is bounded and $N_{f}$ is completely continuous. Since $B, \Pi$ and $\Gamma$ are completely continuous, it follows from (13) that $A_{\lambda}: W^{1, p}(\Omega) \rightarrow\left(W^{1, p}(\Omega)\right)^{*}$ is bounded.

We claim that $A_{\lambda}: W^{1, p}(\Omega) \rightarrow\left(W^{1, p}(\Omega)\right)^{*}$ is a pseudomonotone operator. Let a sequence $\left\{u_{n}\right\} \subset W^{1, p}(\Omega)$ satisfy $u_{n} \rightarrow u$ in $W^{1, p}(\Omega)$ and

$$
\limsup _{n \rightarrow \infty}\left\langle A_{\lambda}\left(u_{n}\right), u_{n}-u\right\rangle \leq 0
$$

The complete continuity of the operators $B, \Pi$ and $\Gamma$ yields the strong convergent sequences $B\left(u_{n}\right) \rightarrow B(u), \Pi\left(u_{n}\right) \rightarrow \Pi(u)$ and $\Gamma\left(u_{n}\right) \rightarrow \Gamma(u)$ in $\left(W^{1, p}(\Omega)\right)^{*}$. This results in

$$
\begin{aligned}
& \lim _{n \rightarrow \infty}\left\langle B\left(u_{n}\right), u_{n}-v\right\rangle=\langle B(u), u-v\rangle, \lim _{n \rightarrow \infty}\left\langle\Pi\left(u_{n}\right), u_{n}-v\right\rangle=\langle\Pi(u), u-v\rangle, \\
& \lim _{n \rightarrow \infty}\left\langle\Gamma\left(u_{n}\right), u_{n}-u\right\rangle=\langle\Gamma(u), u-v\rangle
\end{aligned}
$$

for all $v \in W^{1, p}(\Omega)$. We infer that

$$
\lim _{n \rightarrow \infty}\left\langle B\left(u_{n}\right), u_{n}-u\right\rangle=\lim _{n \rightarrow \infty}\left\langle\Pi\left(u_{n}\right), u_{n}-u\right\rangle=\lim _{n \rightarrow \infty}\left\langle\Gamma\left(u_{n}\right), u_{n}-u\right\rangle=0,
$$

so (15) reduces to

$$
\limsup _{n \rightarrow \infty}\left\langle\tilde{A}\left(u_{n}\right), u_{n}-u\right\rangle \leq 0 .
$$

Inequality (17) enables us to apply Proposition 1 ensuring that the strong convergence $u_{n} \rightarrow u$ in $W^{1, p}(\Omega)$ holds.

At this point, we know that the strong convergence $\nabla\left(u_{n}\right) \rightarrow \nabla(u)$ holds in $\left(L^{p}(\Omega)\right)^{N}$, so the second inequality in $(A 1)$ entails $A\left(\cdot, \nabla u_{n}(\cdot)\right) \rightarrow A(\cdot, \nabla u(\cdot))$ strongly in $\left(L^{p^{\prime}}(\Omega)\right)^{N}$. Then for each $v \in W^{1, p}(\Omega)$ one has

$$
\begin{aligned}
\lim _{n \rightarrow \infty}\left\langle\tilde{A}\left(u_{n}\right), u_{n}-v\right\rangle & =\lim _{n \rightarrow \infty} \int_{\Omega} A\left(x, \nabla u_{n}\right) \cdot \nabla\left(u_{n}-v\right) d x \\
& =\int_{\Omega} A(x, \nabla u) \cdot \nabla(u-v) d x \\
& =\langle\tilde{A}(u), u-v\rangle .
\end{aligned}
$$

Taking into account of (13), (16) and (18), we arrive at

$$
\lim _{n \rightarrow \infty}\left\langle A_{\lambda}\left(u_{n}\right), u_{n}-v\right\rangle=\left\langle A_{\lambda}(u), u-v\right\rangle
$$

for all $v \in W^{1, p}(\Omega)$ and $\lambda>0$. Therefore the operator $A_{\lambda}: W^{1, p}(\Omega) \rightarrow\left(W^{1, p}(\Omega)\right)^{*}$ is pseudomonotone. 
Next we show that the operator $A_{\lambda}: W^{1, p}(\Omega) \rightarrow\left(W^{1, p}(\Omega)\right)^{*}$ is coercive whenever $\lambda>0$ is sufficiently large.

Since $\alpha \in L^{\infty}(\Omega), \alpha \geq 0$, from (11) we note that

$$
\left\langle A_{\lambda}(u), u\right\rangle \geq\langle\tilde{A}(u), u\rangle+\int_{\partial \Omega} \beta(\sigma)|u(\sigma)|^{p} d \sigma+\lambda \int_{\Omega} \pi(x, u) u d x-\int_{\Omega} f(x, T u, \nabla(T u)) u d x
$$

for all $u \in W^{1, p}(\Omega)$. We estimate from below the terms in the right-hand side of (19). Assumption $(A 1)$ and (3) yield

$$
\langle\tilde{A} u, u\rangle \geq c_{1}\|\nabla u\|_{L^{p}(\Omega)}^{p} \text { for all } u \in W^{1, p}(\Omega) .
$$

From (9) we derive that

$$
\int_{\Omega} \pi(x, u(x)) u(x) d x \geq b_{1}\|u\|_{L^{\frac{p}{p-r_{1}}}(\Omega)}^{\frac{p}{p-r_{1}}}-b_{2} \text { for all } u \in W^{1, p}(\Omega),
$$

with positive constants $b_{1}$ and $b_{2}$ (see [3]).

In view of (7), we have that $\underline{u} \leq T u \leq \bar{u}$ a.e. in $\Omega$ whenever $u \in W^{1, p}(\Omega)$. Consequently, we may set $s=(T u)(x)$ in the statement of hypothesis $(H)$. Then, for each $\varepsilon>0$, we obtain through Hölder's and Young's inequalities and the Sobolev embedding theorem the estimate

$$
\begin{aligned}
\left|\int_{\Omega} f(x, T u, \nabla(T u)) u d x\right| & \leq \int_{\Omega}\left(\sigma|u|+a|\nabla(T u)|^{r_{1}}|u|\right) d x \\
& \leq \varepsilon\|\nabla u\|_{L^{p}(\Omega)}^{p}+c(\varepsilon)\|u\|_{L^{\frac{p}{p-r_{1}}}}^{\frac{p}{p-r_{1}}(\Omega)}+d\|u\|_{\beta, 1, p},
\end{aligned}
$$

with positive constants $c(\varepsilon)$ (depending on $\varepsilon$ ) and $d$.

Gathering (19)-(22) leads to

$$
\left\langle A_{\lambda}(u), u\right\rangle \geq\left(c_{1}-\varepsilon\right)\|\nabla u\|_{L^{p}(\Omega)}^{p}+\int_{\partial \Omega} \beta(\sigma)|u(\sigma)|^{p} d \sigma+\left(\lambda b_{1}-c(\varepsilon)\right)\|u\|_{L^{\frac{p}{p-r_{1}}}(\Omega)}^{\frac{p}{p-r_{1}}}-d\|u\|_{\beta, 1, p}-\lambda b_{2}
$$

for all $u \in W^{1, p}(\Omega)$ and $\lambda>0$. Now we fix $\varepsilon$ and $\lambda$ to verify $\varepsilon \in\left(0, c_{1}\right)$ and $\lambda>\frac{c(\varepsilon)}{b_{1}}$. From (2) and (23) it is clear that

$$
\left\langle A_{\lambda}(u), u\right\rangle \geq c_{0}\|u\|_{\beta, 1, p}^{p}-d\|u\|_{\beta, 1, p}-\lambda b_{2}
$$

for all $u \in W^{1, p}(\Omega)$, with a constant $c_{0}>0$. Due to the fact that $p>1$, it turns out

$$
\lim _{\|u\|_{\beta, 1, p} \rightarrow+\infty} \frac{\left\langle A_{\lambda}(u), u\right\rangle}{\|u\|_{\beta, 1, p}}=+\infty,
$$

thereby the operator $A_{\lambda}$ is coercive.

Summarizing, we have proved that the operator $A_{\lambda}: W^{1, p}(\Omega) \rightarrow\left(W^{1, p}(\Omega)\right)^{*}$ is bounded, pseudomonotone and coercive. This allows us to apply Theorem 1 with $\mathcal{A}=A_{\lambda}$ for $\lambda>0$ sufficiently large. The surjectivity of $A_{\lambda}$ implies the existence of a solution $u \in W^{1, p}(\Omega)$ of Equation (14), thus completing the proof.

Remark 1. As a consequence of (23), we can precisely determine the threshold of $\lambda>0$ in the statement of Theorem 2.

\section{Main Abstract Result for Problem (1)}

Our result regarding the method of sub-supersolution for problem (1) is stated as follows. 
Theorem 3. Assume that the conditions $(A 1),(A 2)$ and $(H)$ are satisfied. Then problem $(P)$ possesses a solution $u \in W^{1, p}(\Omega)$ satisfying $\underline{u} \leq u \leq \bar{u}$ a.e. in $\Omega$, where $\underline{u}$ and $\bar{u}$ are the subsolution and the supersolution that are postulated in assumption $(H)$.

Proof. According to Theorem 2 we can fix $\lambda>0$ sufficiently large such that equation (14) admits a solution $u \in W^{1, p}(\Omega)$. Explicitly, this reads as

$$
\begin{aligned}
& \langle\tilde{A}(u), v\rangle+\int_{\Omega} \alpha(x)|u|^{p-2} u v d x+\lambda \int_{\Omega} \pi(x, u) v d x+\int_{\partial \Omega} \beta(x)|u|^{p-2} u v d \sigma \\
& =\int_{\Omega} f(x, T u, \nabla(T u)) v d x \text { for all } v \in W^{1, p}(\Omega) .
\end{aligned}
$$

Let us prove that $u \leq \bar{u}$ a.e. in $\Omega$. Inserting $v=(u-\bar{u})^{+} \in W^{1, p}(\Omega)$ in (6) and (24) renders

$$
\begin{aligned}
& \left\langle\tilde{A}(\bar{u}),(u-\bar{u})^{+}\right\rangle+\int_{\Omega} \alpha(x)|\bar{u}|^{p-2} \bar{u}(u-\bar{u})^{+} d x+\int_{\partial \Omega} \beta(x)|\bar{u}|^{p-2} \bar{u}(u-\bar{u})^{+} d \sigma \\
& \geq \int_{\Omega} f(x, \bar{u}, \nabla \bar{u})(u-\bar{u})^{+} d x
\end{aligned}
$$

and

$$
\begin{aligned}
& \left\langle\tilde{A}(u),(u-\bar{u})^{+}\right\rangle+\int_{\Omega} \alpha(x)|u|^{p-2} u(u-\bar{u})^{+} d x+\lambda \int_{\Omega} \pi(x, u)(u-\bar{u})^{+} d x \\
& +\int_{\partial \Omega} \beta(x)|u|^{p-2} u(u-\bar{u})^{+} d \sigma \\
& =\int_{\Omega} f(x, T u, \nabla(T u))(u-\bar{u})^{+} d x .
\end{aligned}
$$

Subtract (25) from (26) and use (3) and (7) to deduce that

$$
\begin{aligned}
& \int_{\Omega}(A(x, \nabla u)-A(x, \nabla \bar{u})) \nabla(u-\bar{u})^{+} d x+\int_{\partial \Omega} \beta(x)\left(|u|^{p-2} u-|\bar{u}|^{p-2} \bar{u}\right)(u-\bar{u})^{+} d \sigma \\
& +\int_{\Omega} \alpha(x)\left(|u|^{p-2} u-|\bar{u}|^{p-2} \bar{u}\right)(u-\bar{u})^{+} d x+\lambda \int_{\Omega} \pi(x, u)(u-\bar{u})^{+} d x \\
& \leq \int_{\Omega}(f(x, T u, \nabla(T u))-f(x, \bar{u}, \nabla \bar{u}))(u-\bar{u})^{+} d x \\
& =\int_{\{u>\bar{u}\}}(f(x, T u, \nabla(T u))-f(x, \bar{u}, \nabla \bar{u}))(u-\bar{u}) d x=0 .
\end{aligned}
$$

The monotonicity of $A(x, \cdot)$, guaranteed by assumption $\left(A_{2}\right)$, and the monotonicity of the map $\xi \mapsto|\xi|^{p-2} \xi$ on $\mathbb{R}^{N}$ give

$$
\begin{aligned}
& \int_{\Omega}(A(x, \nabla u)-A(x, \nabla \bar{u})) \nabla(u-\bar{u})^{+} d x \\
= & \int_{\{u>\bar{u}\}}(A(x, \nabla u)-A(x, \nabla \bar{u}))(\nabla u-\nabla \bar{u}) d x \geq 0, \\
= & \int_{\Omega} \alpha(x)\left(|u|^{p-2} u-|\bar{u}|^{p-2} \bar{u}\right)(u-\bar{u})^{+} d x \\
& \alpha(x)\left(|u|^{p-2} u-|\bar{u}|^{p-2} \bar{u}\right)(u-\bar{u}) d x \geq 0, \\
= & \int_{\partial \Omega \in \partial \Omega: u>\bar{u}\}} \beta(\sigma)\left(|u|^{p-2} u-|\bar{u}|^{p-2} \bar{u}\right)(u-\bar{u})^{+} d \sigma
\end{aligned}
$$


From (27) and (9) we obtain

$$
\int_{\{u>\bar{u}\}}(u-\bar{u})^{\frac{p}{p-r_{1}}} d x=\int_{\Omega} \pi(x, u)(u-\bar{u})^{+} d x \leq 0,
$$

where $u \leq \bar{u}$ a.e in $\Omega$.

Next we show that $\underline{u} \leq u$ a.e in $\Omega$. Setting $v=(\underline{u}-u)^{+} \in W^{1, p}(\Omega)$ in (5) and (24) produces

$$
\begin{aligned}
& \left\langle\tilde{A}(\underline{u}),(\underline{u}-u)^{+}\right\rangle+\int_{\Omega} \alpha(x)|\underline{u}|^{p-2} \underline{u}(\underline{u}-u)^{+} d x+\int_{\partial \Omega} \beta(x)|\underline{u}|^{p-2} \underline{u}(\underline{u}-u)^{+} d \sigma \\
& \leq \int_{\Omega} f(x, \underline{u}, \nabla \underline{u})(\underline{u}-u)^{+} d x
\end{aligned}
$$

and

$$
\begin{aligned}
& \left\langle\tilde{A}(u),(\underline{u}-u)^{+}\right\rangle+\int_{\Omega} \alpha(x)|u|^{p-2} u(\underline{u}-u)^{+} d x+\lambda \int_{\Omega} \pi(x, u)(\underline{u}-u)^{+} d x \\
& +\int_{\partial \Omega} \beta(x)|u|^{p-2} u(\underline{u}-u)^{+} d \sigma \\
& =\int_{\Omega} f(x, T u, \nabla(T u))(\underline{u}-u)^{+} d x .
\end{aligned}
$$

By subtracting (29) from (28) and taking into account (3) we arrive at

$$
\begin{aligned}
& \int_{\Omega}(A(x, \nabla \underline{u})-A(x, \nabla u)) \nabla(\underline{u}-u)^{+} d x+\int_{\partial \Omega} \beta(x)\left(|\underline{u}|^{p-2} \underline{u}-|u|^{p-2} u\right)(\underline{u}-u)^{+} d \sigma \\
& +\int_{\Omega} \alpha(x)\left(|\underline{u}|^{p-2} \underline{u}-|u|^{p-2} u\right)(\underline{u}-u)^{+} d x-\lambda \int_{\Omega} \pi(x, u)(\underline{u}-u)^{+} d x \\
& \leq \int_{\Omega}\left(f(x, \underline{u}, \nabla \underline{u})-f(x, T u, \nabla(T u))(\underline{u}-u)^{+} d x\right. \\
& =\int_{\{\underline{u}>u\}}(f(x, \underline{u}, \nabla \underline{u})-f(x, T u, \nabla(T u)))(\underline{u}-u)^{+} d x=0 .
\end{aligned}
$$

Along (9) and proceeding as above, (30) results in

$$
-\int_{\{\underline{u}>u\}}-(\underline{u}-u)^{\frac{p}{p-r}} d x=-\int_{\Omega} \pi(x, u)(\underline{u}-u)^{+} d x \leq 0,
$$

which entails that $\underline{u} \leq u$ a.e in $\Omega$, thus proving the claim.

Therefore the solution $u \in W^{1, p}(\Omega)$ of the operator equation (14) verifies the enclosure property $\underline{u} \leq u \leq \bar{u}$ a.e. in $\Omega$. Then we obtain from (7) and (9) that $T u=u$ and $\Pi(u)=0$. Hence for our function $u$ the equalities (24) and (4) coincide. We see that $u \in W^{1, p}(\Omega)$ is a solution of the original problem (1) fulfilling in addition $\underline{u} \leq u \leq \bar{u}$ a.e. in $\Omega$. This completes the proof.

\section{An Application}

The aim of this section is to apply Theorem 3 to establish the existence of positive solutions of Robin problem (1). The main point is to find appropriate ordered sub-supersolutions. The approach can be used to get other types of solutions.

In order to simplify the presentation, we focus on problem (1) driven by the Robin $p$-Laplacian, $1<p<+\infty$, and when $\alpha(x) \equiv 0$ and the $x$-dependence in the convection term $f(x, s, \xi)$ is dropped. We emphasize that $\alpha \equiv 0$ marks a sharp distinction in regard to the Neumann problem. Specifically, we consider the (purely) Robin problem

$$
\begin{cases}-\Delta_{p} u=f(u, \nabla u) & \text { in } \Omega \\ |\nabla u|^{p-2} \nabla u \cdot v(x)+\beta(x)|u|^{p-2} u=0 & \text { on } \partial \Omega,\end{cases}
$$


with $\beta(x) \geq 0$ for a.e. $x \in \partial \Omega, \beta \not \equiv 0$.

We suppose that $f: \mathbb{R} \times \mathbb{R}^{N} \rightarrow \mathbb{R}$ is a continuous function verifying the following assumption:

$\left(H^{\prime}\right)$ There exist constants $a_{0}>0, a_{1}>0, b>0$ and $r_{1} \in\left(0, \frac{p}{\left(p^{*}\right)^{\prime}}\right)$ such that

$$
\begin{gathered}
|f(s, \xi)| \leq a_{1}\left(1+|\xi|^{r_{1}}\right) \text { for all } s \in(0, b], \xi \in \mathbb{R}^{N}, \\
\lambda_{1} s^{p-1} \leq f(s, \xi) \text { for all } s \in\left(0, a_{0}\right),|\xi|<a_{0}
\end{gathered}
$$

and

$$
f(b, 0)=0 .
$$

The condition (33) involves the first eigenvalue $\lambda_{1}$ of the (negative) Dirichlet $p$-Laplacian as given in (8). Let us note that $u=b$ is not a solution to problem (31) because the boundary condition is not verified. We formulate the following result concerning problem (31).

Theorem 4. Assume that the conditions $(A 1),(A 2)$ and $\left(H^{\prime}\right)$ are satisfied. Then the Robin problem (31) possesses a (positive) solution $u \in W^{1, p}(\Omega)$ satisfying $0<u \leq b$ a.e. in $\Omega$.

Proof. Fix an eigenfunction $\varphi_{1}$ of $-\Delta_{p}$ on $W_{0}^{1, p}(\Omega)$, with $\varphi_{1}>0$ in $\Omega$, corresponding to the first eigenvalue $\lambda_{1}$ (see (8) and the related comments). Since $\varphi_{1} \in C^{1}(\bar{\Omega})$, we can choose an $\varepsilon>0$ such that

$$
\varepsilon \varphi_{1}(x)<a_{0} \text { and } \varepsilon\left|\nabla \varphi_{1}(x)\right|<a_{0} \text { for all } x \in \Omega,
$$

where $a_{0}$ is the positive constant prescribed in hypothesis $\left(H^{\prime}\right)$.

We note that $\underline{u}=\varepsilon \varphi_{1}$ is a subsolution in the sense of (5) for the Robin problem (31). Indeed, by (8), (33) and (35) and since the trace of $\underline{u}$ on $\partial \Omega$ vanishes, we infer that

$$
\begin{aligned}
\int_{\Omega}|\nabla \underline{u}|^{p-2} \nabla \underline{u} \cdot \nabla v d x+\int_{\partial \Omega} \beta(x)|\underline{u}|^{p-2} \underline{u} v d \sigma & =\varepsilon^{p-1} \int_{\Omega}\left|\nabla \varphi_{1}(x)\right|^{p-2} \nabla \varphi_{1}(x) \cdot \nabla v(x) d x \\
& =\lambda_{1} \int_{\Omega}\left(\varepsilon \varphi_{1}(x)\right)^{p-1} v(x) d x \\
& \leq \int_{\Omega} f\left(\varepsilon \varphi_{1}(x), \varepsilon \nabla \varphi_{1}(x)\right) v(x) d x \\
& =\int_{\Omega} f(\underline{u}(x), \nabla \underline{u}(x)) v(x) d x \text { for all } v \in W^{1, p}(\Omega), v \geq 0 .
\end{aligned}
$$

This proves that $\underline{u}=\varepsilon \varphi_{1}$ is a subsolution of problem (31).

Now we observe that the constant function $\bar{u}=b$ is a supersolution of problem (31). Indeed, let us notice from assumption (34) that

$$
\begin{aligned}
\int_{\Omega}|\nabla \bar{u}|^{p-2} \nabla \bar{u} \cdot \nabla v d x+\int_{\partial \Omega} \beta(x)|\bar{u}|^{p-2} \bar{u} v d \sigma & =\int_{\partial \Omega} \beta(x) b^{p-1} v(x) d \sigma \\
& \geq 0=\int_{\Omega} f(b, 0) v(x) d x \\
& =\int_{\Omega} f(\bar{u}(x), \nabla \bar{u}(x)) v(x) d x
\end{aligned}
$$

for all $v \in W^{1, p}(\Omega)$ with $v \geq 0$, which confirms that $\bar{u}=b$ is a supersolution of problem (31) in the sense of (6).

For a possibly smaller $\varepsilon>0$ to be fulfilled $\varepsilon \varphi_{1}(x) \leq b$ whenever $x \in \Omega$, the inequality $\underline{u} \leq \bar{u}$ holds true. The growth condition in $(H)$ is satisfied due to (32) because the pointwise intervals $[\underline{u}(x), \bar{u}(x)]$ are all included in the bounded interval $(0, b]$. Altogether we are in a position to apply Theorem 3 , which yields the desired conclusion.

We provide a simple example illustrating the applicability of Theorem 4 . 
Example 1. Let $f: \mathbb{R} \times \mathbb{R}^{N} \rightarrow \mathbb{R}$ be defined by

$$
f(s, \xi)=g(s)+h(\xi) \text { for all }(s, \xi) \in \mathbb{R} \times \mathbb{R}^{N},
$$

with $g: \mathbb{R} \rightarrow \mathbb{R}$ defined by

$$
g(s)=\left\{\begin{array}{lr}
0 & \text { if } s<0 \text { or } s>2 \\
\lambda_{1} s^{p-1} & \text { if } 0 \leq s \leq 1 \\
\lambda_{1}(2-s)^{p-1} & \text { if } 1<s \leq 2
\end{array}\right.
$$

and any continuous function $h: \mathbb{R}^{N} \rightarrow \mathbb{R}$ satisfying $h(\xi) \geq 0, h(0)=0$ and

$$
0 \leq h(\xi) \leq a_{2}\left(1+|\xi|^{r_{1}}\right) \text { for all } \xi \in \mathbb{R}^{N},
$$

with constants $a_{2}>0$ and $r_{1} \in\left(0, \frac{p}{\left(p^{*}\right)^{\prime}}\right)$. We note that $f(2,0)=0$ and

$$
f(s, \xi)=g(s)+h(\xi) \geq \lambda_{1} s^{p-1} \text { for all } 0 \leq s \leq 1, \xi \in \mathbb{R}^{N} .
$$

Hypothesis $\left(H^{\prime}\right)$ is verified taking $a_{0}=1$ and $b=2$. Theorem 4 can be applied to problem (31) with $f(s, \xi)$ given above.

Author Contributions: Conceptualization, D.M., A.S. and E.T. All authors contributed equally to this paper. All authors have read and agreed to the published version of the manuscript.

Funding: This research received no external funding.

Acknowledgments: The last two authors are members of Gruppo Nazionale per l'Analisi Matematica, la Probabilità e le loro Applicazioni (GNAMPA) of Istituto Nazionale di Alta Matematica (INdAM). The paper is partially supported by PRIN 2017-Progetti di Ricerca di rilevante Interesse Nazionale, Nonlinear Differential Problems via Variational, Topological and Set-valued Methods. The authors thank the referees for careful reading and useful comments that helped to improve the paper.

Conflicts of Interest: The authors declare no conflict of interest.

\section{References}

1. Motreanu, D.; Motreanu, V.V.; Papageorgiou, N.S. Topological and Variational Methods with Applications to Nonlinear Boundary Value Problems; Springer: New York, NY, USA, 2014.

2. Miyajima, S.; Motreanu, D.; Tanaka, M. Multiple existence results of solutions for the Neumann problems via super- and sub-solutions. J. Funct. Anal. 2012, 262, 1921-1953. [CrossRef]

3. Motreanu, D.; Sciammetta, A.; Tornatore, E. A sub-supersolution approach for Neumann boundary value problems with gradient dependence. Nonlinear Anal. Real World Appl. 2020, 54, 103096. [CrossRef]

4. Averna, D.; Papageorgiou, N.S.; Tornatore, E. Positive solutions for nonlinear Robin problems with convection. Math. Methods Appl. Sci. 2019, 42, 1907-1920. [CrossRef]

5. Bai, Y.; Gasinski, L.; Papageorgiou, N.S. Nonlinear nonhomogeneous Robin problems with dependence on the gradient. Bound. Value Probl. 2018, 17, 1-24. [CrossRef]

6. Candito, P.; Gasinski, L.; Papageorgiou, N.S. Nonlinear nonhomogeneous Robin problems with convection. Ann. Acad. Sci. Fenn. Math. 2019, 44, 755-767. [CrossRef]

7. Faraci, F.; Motreanu, D.; Puglisi, D. Positive solutions of quasi-linear elliptic equations with dependence on the gradient. Calc. Var. Partial Differ. Equ. 2015, 54, 525-538. [CrossRef]

8. Papageorgiou, N.S.; Radulescu, V.; Repovs, D. Positive solutions for nonvariational Robin problems. Asymptot. Anal. 2018, 108, 243-255. [CrossRef]

9. Guarnotta, U.; Marano, S.A.; Motreanu, D. On a Singular Robin Problem with Convection Terms. Available online: http:/ /arxiv.org/abs/1909.09834 (accessed on 1 March 2020).

10. Averna, D.; Papageorgiou, N.S.; Tornatore, E. Positive solutions for nonlinear Robin problems. Electron. J. Differ. Equ. 2017, 204, 1-25. [CrossRef] 
11. D'Aguì, G.; Marano, S.; Papageorgiou, N.S. Multiple solutions to a Robin problem with indefinite weight and asymmetric reaction. J. Math. Anal. Appl. 2016, 433, 1821-1845. [CrossRef]

12. Guarnotta, U.; Marano, S.A.; Papageorgiou, N.S. Multiple nodal solutions to a Robin problem with sign-changing potential and locally defined reaction. Atti Accad. Naz. Lincei Rend. Lincei Mat. Appl. 2019, 30, 269-294. [CrossRef]

13. Marano, S.A.; Papageorgiou, N.S. Asymmetric Robin boundary-value problems with p-Laplacian and indefinite potential. Electron. J. Differ. Equ. 2018, 2018, 1-21.

14. Papageorgiou, N.S.; Radulescu, V.; Repovs, D. Positive solutions for perturbations of the Robin eigenvalue problem plus an indefinite potential. Discret. Contin. Dyn. Syst. 2017, 37, 2589-2618. [CrossRef]

15. Papageorgiou, N.S.; Winkert, P. Solutions with sign information for nonlinear nonhomogeneous problems. Math. Nachr. 2019, 292, 871-891. [CrossRef]

16. Faria, L.; Miyagaki, O.; Motreanu, D. Comparison and positive solutions for problems with (p,q)-Laplacian and convection term. Proc. Edinb. Math. Soc. 2014, 57, 687-698. [CrossRef]

17. Fragnelli, G.; Mugnai, D.; Papageorgiou, N.S. Robin problems for the p-Laplacian with gradient dependence. Discret. Contin. Dyn. Syst. Ser. S 2019, 12, 287-295. [CrossRef]

18. De Araujo, A.; Faria, L. Positive solutions of quasilinear elliptic equations with exponential nonlinearity combined with convection term. J. Differ. Equ. 2019, 267, 4589-4608. [CrossRef]

19. Gilbarg D.; Trudinger, N. Elliptic Partial Differential Equations of Second Order; Reprint of the 1998 edition, Classics in Mathematics; Springer: Berlin, Germany, 2001.

20. Ladyzhenskaya, O.; Uraltseva, N. Linear and Quasilinear Elliptic Equations; Academic Press: New York, NY, USA; London, UK, 1968.

21. Motreanu, D.; Tornatore, E. Location of solutions for quasilinear elliptic equations with gradient dependence. Electron. J. Qual. Theory Differ. Equ. 2017, 87, 1-10. [CrossRef]

22. Carl, S.; Le, V.K.; Motreanu, D. Nonsmooth Variational Problems and Their Inequalities. Comparison Principles and Applications; Springer: New York, NY, USA, 2007.

23. Colasuonno, F. ; Pucci, P.; Varga, C. Multiple solutions for an eigenvalue problem involving p-Laplacian type operator. Nonlinear Anal. 2012, 75, 4496-4512. [CrossRef]

(C) 2020 by the authors. Licensee MDPI, Basel, Switzerland. This article is an open access article distributed under the terms and conditions of the Creative Commons Attribution (CC BY) license (http:/ / creativecommons.org/licenses/by/4.0/). 